\title{
Retrieval of attribute information as a function of processing depth
}

\author{
MARK A. DORFMUELLER and DONALD A. SCHUMSKY \\ University of Cincinnati, Cincinnati, Ohio 45221
}

\begin{abstract}
The effects of processing depth on retrieval of attribute information was investigated using a modified probe-recognition procedure. Subjects decided whether a probe word was a synonym, a homonym, identical to, or not related to any word of a previously presented threeword set. Depth of processing was manipulated by priming with a dimensional cue prior to the presentation of the study set. The dimensional cue specified the relation in question between probes and potential target words in each study set. Effects were assessed relative to unprimed conditions where subjects had the dimensional relation specified during probe presentation. The probe word followed the three-word set by a 0-, 10-, or 30-sec filled or unfilled delay. Priming effects were reflected in reaction time but not recognition accuracy, with degree of effect related to level of processing. Synonyms were affected most, homonyms next, identity least.
\end{abstract}

Craik and Lockhart (1972) proposed a levels-ofprocessing view of memory, asserting that performance on a recall or recognition task is a positive function of the depth to which stimuli are processed. Shallower depths of processing represent the analysis of physical or sensory properties (structural attributes) of stimuli and deeper stages the extraction of meaning (semantic attributes). Support for the levels-of-processing view has been obtained employing a variety of stimuli in both recognition and recall tasks (Bower \& Karlin, 1974; Craik \& Tulving, 1975; Gardiner, 1974; Nelson, Wheeler, Borden, \& Brooks, 1974; Till \& Jenkins, 1973; Kimble, Schumsky, \& Warm, Note 1). However, all of these studies have focused upon memory for the to-be-remembered (TBR) stimulus per se. That is, previous studies have investigated whether retention of a TBR stimulus is related to the level to which the stimulus is processed.

The focus of the present study is on retention of attribute information. Specifically, one may question whether the availability of attribute information encoded is related to the level at which stimuli are processed. That is, whether, in addition to facilitating the accessing of the TBR stimulus, access to stimulusattribute information is facilitated as well. Thus, if shallower depths of processing involve the analysis, elaboration, and storage of sensory properties, and deeper levels involve the analysis, elaboration, and storage of semantic information, then altered access to these elaborated properties should be demonstrable.

One means of assessing the availability of attribute information is via a technique known as a modified

This article is based on a seniar thesis completed by the first author under the direction of the second. Portions of this research were presented at the Midwestern Psychological Association Annual Meeting, Chicago, May 1976. Reprint requests should be sent to Donald A. Schumsky, Department of Psychology, University of Cincinnati, Cincinnati, Ohio 45221. probe-recognition procedure. In such a procedure, subjects are presented a list of words followed by a probe stimulus that may be a synonym, a homonym, or identical to one of the words in the acquisition list. On any particular trial, subjects are required to make a recognition judgment on the basis of one of the three similarity criteria. Using such a procedure, Shulman (1970) found that recognition accuracy with homonym and identical probe stimuli was better and reaction times faster than with synonym probe stimuli.

In the Shulman (1970) study, however, the cue specifying the similarity criterion for a particular trial was presented immediately prior to the occurrence of the probe word. Thus, encoding of the acquisition list on any particular trial was presumably uninfluenced by the nature of the recognition probe type. The usual orienting task in a levels-of-processing experiment might be viewed as priming subjects for certain encoding dimensions. Thus, in the present experiment, an attempt was made to prime semantic and phonemic attributes of words by informing the subject of the dimensional nature of a later probe-recognition test prior to encoding the acquisition list and by instructions to attend to appropriate stimulus attributes. Effects of priming on recognition performance were assessed relative to nonprimed conditions, that is, conditions analogous to Shulman's (1970) study. Cuing on the basis of homophony or identity was expected to orient the subject's encoding toward the relevant, "shallower" sensory attributes, while cuing on the basis of synonymy was expected to result in deeper levels of processing. Thus, the present experiment attends to the availability of attributes. That is, does priming for a given level affect the availability of the appropriate attribute information?

A second aspect of the present experiment was to evaluate the effects of priming on reaction time. Previous studies have employed reaction time measures of en- 
coding time as an index of levels of processing (Craik \& Tulving, 1975; Gardiner, 1974). In the present experiment, reaction time was taken at retrieval. It might be expected that retrieval time should vary systematically as a function of depth and, as a consequence, covary with recognition performance. As with recognition performance, the effects of priming on reaction time were assessed relative to nonprimed conditions.

Finally, if the effects of priming take processing time and space, then an unfilled delay between target-set presentation and recognition test should facilitate recognition performance relative to a filled-cielay test. Therefore, it was expected that recognition accuracy would be poorer in filled-delay than in unfilled-delay conditions, and that recognition performance in filled conditions would decrease as the duration of the retention interval increased (e.g., Brown, 1958; Peterson \& Peterson, 1959).

\section{METHOD}

Design

A 2 (within-subjects) by 2 (between-subjects) design was employed. Factorial combination of two levels of dimensional cue position (primed, i.e., the cue was presented prior to a three-word target set, vs. nonprimed, i.e., the cue was presented simultaneously with a probe word) and two levels of delay type (filled vs. unfilled delay) resulted in four experimental conditions $(n=18)$. The running order of the experimental conditions was randomized. Within-subjects factors were dimensional cue type (synonym vs. homonym vs. identical) and retention interval $(0,10$, or $30 \mathrm{sec})$. Levels of dimensional cue type were factorially combined with levels of retention interval.

Each subject received 9 practice and 108 experimental trials. The nature of a typical trial depended on the experimental condition to which a subject was assigned. At the onset of each trial, all subjects were presented with a "Ready" slide for $6 \mathrm{sec}$. For the primed conditions, the second slide consisted of a 2-sec presentation of one of three abbreviated dimensional cues: "syn" (synonym), "hom" (homonym), or "ident" (identical). The cue specified to the subject the dimension of similarity for matching probe to target stimulus on that trial. Subjects in the nonprimed conditions were presented a blank slide in place of the prime. The third slide on every trial for all subjects consisted of the presentation of a three-word study set for $6 \mathrm{sec}$. The next slide, for the filled-delay conditions, was a three-digit number from which the subjects were required to count backward by threes for the duration of the retention interval. For the unfilled-delay conditions, a blank slide was presented in place of the number slide. Finally, a dimensional cue/probe-word slide was presented. For nonprimed conditions, the cue served to indicate to the subjects the recognition criterion for that particular trial, while for the primed conditions, the cue served as a reminder.

The 108 experimental trials consisted of 36 trials with each type of dimensional cue, 12 trials at each of three retention intervals. Three of each of the 12 trials were "no" response, catch trials. The remaining 9 trials required a "yes" response. The 108 trials were divided into three consecutive 36-trial blocks, each containing three observations and 1 catch trial from every combination of dimensional cue type and retention interval. The occurrence of any particular dimensional cue, retention interval, and position of the target word in the study set was randomized, with runs restricted to 3 consecutive trials. The resulting order of presentation of the three blocks of trials was then balanced between and within experimental conditions.

\section{Materials}

A total of 439 monosyllabic, disyllabic, or trisyllabic words from three to six letters in length, occurring at least once per million in the Thorndike-Lorge (1944) general count were selected. Twenty-nine synonym pairs were selected from Webster's New Dictionary of Synonyms (1973). Examples of synonym pairs are adhere-stick, mimic-copy, and origin-source. Twenty-nine homonym pairs were selected from Whitford (1966). Some homonym-pair examples are: doe-dough, earn-urn, and pedal-peddle. Twenty-nine single words were chosen for the identical pairs, plus an additional 294 words to serve as filler items and catch-trial probe words. The distributions of word lengths and Thorndike-Lorge frequencies for synonym, homonym, and identical pairs were made as nearly equal as possible.

One member of each pair of the synonym, homonym, identical, or unrelated word pairs was assigned two filler words, while the remaining member served as the probe stimulus for that particular study set. For all pairs, care was taken to insure that neither of the filler items was related semantically or phonetically to either member. For the homonym pairs, in addition to the above restriction, filler words were chosen such that each filler word also had a potential homonym. This was done in order to control for the apparent salience of the homonym target word in primed conditions. Pilot work indicated that in primed conditions, filler words that did not have a potential homonym were inappropriate distractor items. One example of a homonym probe stimulus and corresponding study set is: yew, hole; ewe, pact.

As an additional check for any ambiguities that might have been present, a group of five graduate students was given the list of 117 probe stimuli and their corresponding study sets in booklet form. The subjects were instructed that the probe stimulus could be a synonym or a homonym, or identical to one of the words in the target set, or unrelated to all of the words in the target set. Given only this information, the subjects' task was to determine which one of the three words in the corresponding study set was related to the probe stimulus or if none of the words were related to the probe stimulus. The task was selfpaced. Mean number of errors was quite small (mean $=1.6)$ and no two subjects made an error on the same word set.

The position of the target word, that is the synonym, homonym, or identical pair member in the study set, was balanced such that each type of target word occurred equally of ten in each of the three possible positions (first, middle, or last) in the study set. No two words in any of the sets began with the same first letter.

\section{Procedure}

The subject was seated on a table-arm chair in front of a projection screen in a dimly lit room. On the arm was a pushbutton response box. One key of the response box was labeled "yes" and the other "no." The response box was connected to a control unit consisting of five interval timers, a Kodak Carousel projector, and a Standard electric timer. The control unit turned on the electric clock simultaneously with the presentation of the cue/probe stimulus. Depression of a response key by the subject stopped the electric clock, at which point the experimenter recorded the subject's response ("yes" or "no") and response time. Half the subjects responded "yes" by depression of the "yes" with the left hand and half with the right hand. All slide presentation intervals were controlled by the interval timers, except the duration of the cue/probe-stimulus slide, which remained on until the subject responded. Immediately following the subject's response, the next trial began.

Prior to the 108 experimental trials, the subject was presented 9 practice trials to familiarize him with the task. Subjects were run individually, and an experimental session lasted approximately $1 \mathrm{~h}$ and $45 \mathrm{~min}$. The subjects were given no feedback concerning their performance during the experiment. 


\section{Instructions}

For nonprimed conditions, instructions emphasized the unpredictable nature of the series of trials with respect to the similarity criteria (i.e., synonymy, homophony, or identity) and prepared the subjects to be tested on any of the three possible dimensions. For primed conditions, instructions emphasized attending to the dimension specified by the cue while trying to memorize the three-word target set for that trial. For all conditions, instructions emphasized both speed of response and accuracy.

\section{Subjects}

The subjects were 77 undergraduate students. Their participation fulfilled a requirement for an introductory psychology course at the University of Cincinnati. Data from five subjects were discarded due to failure to follow instructions or to malfunctioning equipment. Subjects were randomly assigned to an experimental condition upon entering the laboratory.

\section{RESULTS}

Data were separated into four categories corresponding to correct and incorrect "yes" and "no" responses. Separate analyses were performed on recognition accuracy and reaction time on correct "yes" and "no" trials only. A 2 (within-subjects) by 2 (between-subjects) analysis of variance was performed separately on the arcsintransformed percentage scores and median reaction times.

There was little evidence that priming enhanced recognition. Effects of dimensional cue position and the interaction between dimensional cue position and probe type were not significant $(\mathrm{Fs}<1.00)$. The probe-type main effect, however, was significant $[F(2,136)=182.09$, $\mathrm{p}<.01]$; better recognition performance was obtained with identical and homonym probes than with synonym probes. This last result is consistent with that obtained by Shulman (1970). The comparable sources of variance with the correct "no" data all failed to reach significance.

Response times for correct "yes" responses, for each attribute in both primed and nonprimed conditions, are presented in Figure 1. Although priming had no effect on recognition accuracy, the data in Figure 1 indicate that reaction times were faster in primed conditions and differentially so as a function of probe type. The main effects of dimensional cue position $[F(1,68)=29.72]$ and probe type $[F(2,136)=68.59]$, as well as their interaction $[\mathrm{F}(2,136)=8.85]$, were all significant at the $1 \%$ level of confidence. As can be seen from Figure 1, the greatest difference between primed and nonprimed conditions accrued with synonyms, next with homonyms, and least with identical probe stimuli. Similar results were obtained with the analyses of reaction times for correct "no" trials. Again, main effects of dimensional cue position $[F(1,68)=36.02]$ and probe type $[F(2,136)=68.59]$, as well as their interaction $[\mathrm{F}(2,136)=8.85]$, were all significant at the $1 \%$ level of confidence.

It is clear from the above results that priming facilitated retrieval time but not recognition accuracy. Moreover, the finding that priming differentially affects reaction time as a function of probe type discounts the

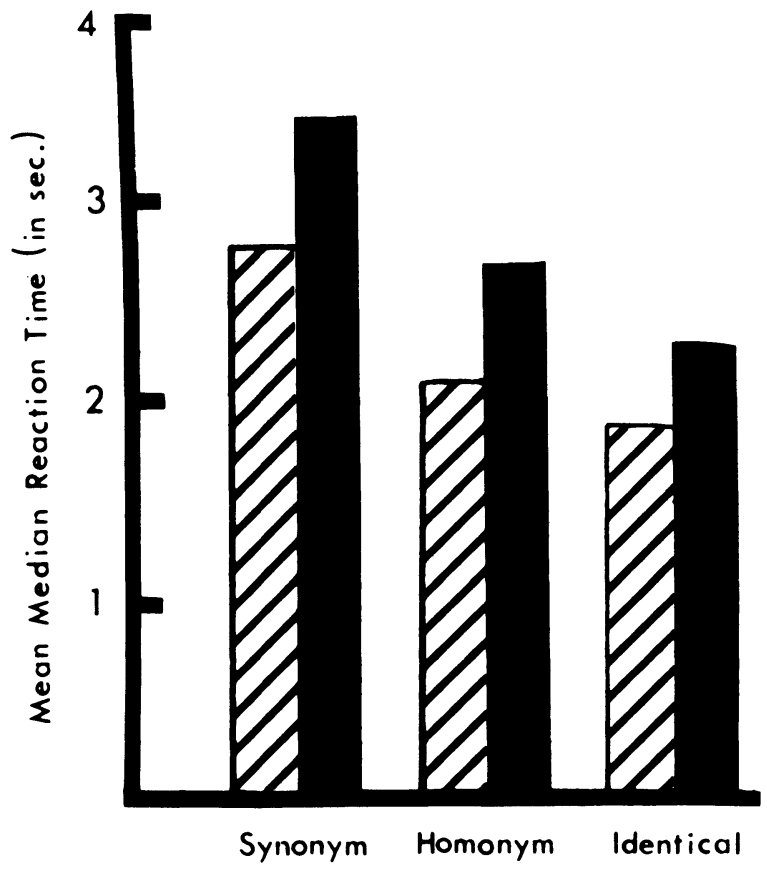

Figure 1. Mean median reaction times of primed (striped bars) and nonprimed (black bars) conditions as a function of probe type.

possibility that reaction times in nonprimed conditions were slower due only to the fact that subjects were required to note the cue in addition to the probe stimulus.

The above results suggest that priming affects initial processing of the acquisition list, which in turn influences retrieval time. Additional support for this argument is provided by the results of the analysis of filled vs. unfilled delays as a function of cue position. Reaction times were significantly faster with unfilled delays for primed conditions than for nonprimed conditions $[F(1,68)=13.86, p<.01]$. The main effect of delay type on reaction time was not significant. With regard to recognition performance, filled delays resulted in significantly poorer performance than did unfilled delays $[F(1,68)=27.12, p<.01]$. The interaction between priming and delay type, however, failed to reach significance.

A Tukey honestly significant difference test performed on the appropriate means revealed that unfilled compared to filled delays produced a significant decrease in reaction time for primed conditions $(p<.01)$ but not for nonprimed conditions. Of further interest is the finding that there was no significant difference in mean reaction time for filled delays; however, unfilled delays in primed conditions were significantly faster than unfilled delays in nonprimed conditions $(p<.01)$. Analysis of delay type as a function of probe type suggests that the greatest effect of unfilled delays on reaction time accrued with synonym probe stimuli. The interaction between delay type and probe type was significant $[F(2,136)=4.59, \mathrm{p}<.05]$.

Finally, the effects of delay type as a function of re- 
tention interval on recognition performance $[\mathrm{F}(2,136)=$ $9.12, \mathrm{p}<.01]$ and response time $[\mathrm{F}(2,136)=8.41$, $\mathrm{p}<.01]$ were statistically significant. The main effect of retention interval on these two measures also reached significance $[F(2,136)=12.31, p<.01$, and $F(2,136)=$ $9.12, \mathrm{p}<.01$, respectively]. As expected in the filleddelay condition, recognition performance decreased and response time increased as the retention interval increased. With unfilled delays, recognition performance and response time remained essentially constant across retention intervals.

\section{DISCUSSION}

Priming in the present experiment had little effect on recognition accuracy. Primed and nonprimed conditions did not differ significantly in recognition, nor was there any significant variation between primed and nonprimed conditions as a function of probe type. On the other hand, priming did significantly reduce reaction times. Moreover, response times were differentially reduced as a function of probe-type level.

One explanation for the present failure to enhance recognition performance by priming is that subjects in the primed conditions simply ignore the dimensional cue. This is unlikely, since in the present experiment priming yielded reaction time differences. Yet it still may be argued that the presentation of dimensional cues in the present experiment failed to produce variations in processing depth. That is, the use of dimensional cues is not the typical manipulation employed to produce the levels-ofprocessing effect. Such an argument, however, seems to be contraindicated by the finding that reaction times were differentially speeded by priming as a function of probe type. In the present study, in regard to reaction time, the least enhancement due to priming was seen with the identical (physical) match, next with the homonym (phonemic) match, and the greatest enhancement with the synonym (semantic) match. This pattern of results is in close agreement with what one might expect according to a levels-of-processing view. Thus, it would appear that dimensional cues effectively oriented the subject's analysis.

A possible alternative explanation suggests itself when the nature of the retention test is considered. In all of the previous experiments demonstrating a levels-of-processing effect, retention was assessed by the subject's retrieval of the primed item. In the present experiment, retention was based on matching a probe to a target stimulus on the basis of a common attribute. Our results suggest that levels-of-processing encoding may enhance retention of the item itself and not increase retrieval accuracy for attribute information. The present results suggest, in addition, that the effects of priming take processing time and space. That is, attentional interference (a filled delay) eliminates the effect of priming on response time. Thus, it would appear that priming influences the rate of initial processing of the acquisition list.

Finally, it is important to mention that the results of the present study are consonant with those obtained by Shulman (1970). The present experiment replicates Shulman's demonstration of the availability of semantic codes in short-term memory.
Both here and in the Shulman study, homonym and identical probe stimuli were recognized better and responded to faster than synonym probe stimuli. In addition, Baddeley (1972) has questioned Shulman's conclusion that his study offers evidence for the presence of semantic coding in short-term memory. Baddeley offers an alternative interpretation of the Shulman finding based upon retrieval processes. It would seem that he would be hard pressed to provide such a retrieval account of the present data.

\section{REFERENCE NOTE}

1. Kimble, G., Schumsky, D. A., \& Warm, J. S. Memory for form: A level-of-processing approach. Paper presented at MPA Annual Meeting, Chicago, May 1976.

\section{REFERENCES}

Baddeley, A. D. Retrieval rules and semantic coding in short-term memory. Psychological Bulletin, 1972, 78, 379-385.

Bower, C. H., \& Karlin, M. B. Depth of processing pictures of faces and recognition memory. Journal of Experimental Psychology, 1974, 103, 751-757.

Brown, J. A. Some tests of the decay theory of immediate memory. Quarterly Journal of Experimental Psychology, 1958, 10, 12-21.

CraIk, F. I. M., \& LockharT, R. S. Levels of processing: A framework for memory research. Journal of Verbal Learning and Verbal Behavior, 1972, 11, 671-684.

Craik, F. I. M., \& Tulving, E. Depth of processing and the retention of words in episodic memory. Journal of Experimental Psychology: General, 1975, 104, 268-294.

GARDINER, J. M. Levels of processing in word recognition and subsequent free recall. Journal of Experimental Psychology, 1974, 102, 101-105.

Nelson, D. L., Wheeler, J. W., JR., Borden, R. D., \& BRooks, D. H. Levels of processing and cueing: Sensory versus meaning features. Journal of Experimental Psychology, 1974, 103, 971-977.

Peterson, L. R., \& Peterson, M. J. Short term retention of individual items. Journal of Experimental Psychology, 1959, 58, 193-198.

Shulman, H. G. Encoding and retention of semantic and phonemic information in short-term memory. Journal of Verbal Learning and Verbal Behavior, 1970, 9, 499-508.

ThoRndike, E. L., \& LORGE, I. The teacher's word book of 30,000 words. New York: Teacher's College Press, 1944.

TILl, R. E., \& Jenkins, J. J. The effects of cued orienting tasks on the free recall of words. Journal of Verbal Learning and Verbal Behavior, 1973, 12, 489-498.

WEBSTER's NEW DictionARY OF SYNONYMS. Springfield, Mass: Merriam, 1973.

WhITfORD, H. C. A dictionary of American homophones and homographs. New York: Teacher's College Press, 1966.

(Received for publication July 6, 1978.) 